\title{
SWAC COMPUTES 126 DISTINCT SEMIGROUPS OF ORDER 4
}

\author{
GEORGE E. FORSYTHE ${ }^{1}$
}

1. Summary and history. A semigroup is a set of elements closed under an associative multiplication. Two semigroups are here considered distinct if they are neither isomorphic nor anti-isomorphic. Let $f(n)$ be the number of distinct semigroups of order $n$. Prior work $[1 ; 5]$ shows that $f(1)=1, f(2)=4, f(3)=18$. For $n=4$, Poole [3] lists 55 distinct commutative semigroups. Carman, Harden, and Posey [1] correct some errors in [3], add 66 distinct noncommutative semigroups for $n=4$, and list all 121 semigroups.

The present work achieves three results believed to be new:

A. The total number of semigroups of order 4, including isomorphs and anti-isomorphs, is found to be 3492. Of these, 126 are distinct, and 58 are distinct and commutative.

Thus $f(4)$ turns out to be 126 .

B. The author has on punched cards multiplication tables for all 3492 semigroups of order 4.

C. The table below includes multiplication tables for all 126 distinct semigroups of order 4.

The table verifies that all 121 semigroups of [1] are correct, and adds five new ones. After the list was prepared, Professor Takayuki Tamura wrote Professor Hewitt that he also has prepared a list of distinct semigroups of order $4 .^{2}$

2. The computation. The principal computing tool used here is SWAC, a fast electronic computer [2]. Any multiplication table on four elements (taken to be $0,1,2,3$, for machine reasons) is considered to have its four rows arranged consecutively; see the table. This permits two binary digits to represent each element, and allows the codes to make efficient use of SWAC's "carry" and "overflow" functions. Each distinct semigroup is represented by its normal form - the first in lexicographic order among the multiplication tables of its isomorphs and anti-isomorphs.

The SWAC computation is sequenced by four codes written by the author for this problem. None of these requires use of SWAC's

Presented to the Society, June 19, 1954; received by the editors July 28, 1954.

${ }^{1}$ Work performed at the National Bureau of Standards, Los Angeles, under sponsorship of the Office of Naval Research. The author wishes to thank Professor Edwin Hewitt for suggesting the computation and furnishing references, and Mr. J. L. Selfridge for help in code checking and preparing the tables.

2 See note at end of article. 
magnetic drum. The four codes have been filed for public examination with: (i) Librarian, Numerical Analysis Research, University of California, Los Angeles 24, Calif., and (ii) Computation Laboratory, National Bureau of Standards, Washington 25, D. C.

The first code causes SWAC to examine in lexicographic order the $4^{16}$ (about $4 \times 10^{9}$ ) possible multiplication tables for four elements, and to punch out the associative ones. The associativity test takes SWAC about $10^{-1}$ second per table; at this rate it would require over 13 years for $4^{16}$ tests. Testing of partial tables for associativity, however, allows SWAC to reject large blocks of tables at a time, and to punch out all 3492 semigroups in less than 80 minutes.

The second code causes SWAC to punch out the normal form of each of the 3492 multiplication tables - a mostly unnecessary procedure consuming about 120 minutes.

The third code causes SWAC to reject the duplicates among the 3492 normal forms, and punch out the 126 distinct normal forms in lexicographic order. It runs in 5 to 10 minutes.

The fourth code converts the 126 normal forms from base 2 notation to base 4 , and punches each normal form on a separate card for later listing on a tabulator.

Each code has been run twice on different days and the outputs are identical.

3. Table of distinct semigroups. The normal forms (see $\$ 2$ ) of the distinct semigroups of orders $n=2,3$, and 4 are given in the table below. The table contains the following information for each distinct semigroup: $N$, its number in the lexicographic order of the normal forms; $C$, the number of the corresponding semigroup in the list of [1]; $R_{i}$, the $i$ th row of its multiplication table in normal form $(i=1, \cdots, n)$.

The multiplication tables for $n=3$ were normalized by J. L. Selfridge and T. S. Motzkin from those in [5]. The normal forms for $n=4$ are a reproduction of the output of the fourth SWAC code described above.

The author believes the table for $n=4$ to be correct and complete for the following reasons: (a) the normal forms were generated twice on SWAC from codes the author prepared personally, and the printer's copy was tabulated directly from SWAC's punch-card output; (b) a machine computation shows that 121 of the semigroups in the table are isomorphs or anti-isomorphs of the 121 semigroups of [1], while the five semigroups not in [1] have been checked by hand for associativity. Ordinarily coding mistakes, if present, result in enormous errors. 
Note added in proof. Professor Tamura's list (see $\$ 1$ above) has now appeared in the Journal of Gakugei, Tokushima Univ. vol. 5 (1954) pp. 17-27. It was computed by hand by Tamura and M. Yamamura in 1953 and 1954, and was submitted for publication in August 1954. Using the second and third SWAC codes (see §2), the author finds that Tamura's 126 semigroups are (up to isomorphism or anti-isomorphism) precisely those generated by SWAC.

The correspondence with Tamura's list can be established from the following table, giving Tamura's numbers for the 126 semigroups in the order of my table below:

$0-001,003,118,010,004,078,079,030,044,009$,
$1-006,002,119,031,053,008,117,007,043,005$,
$2-101,071,075,110,093,080,063,108,077,038$,
$3-083,061,026,025,049,039,045,076,036,120$,
$4-054,121,116,100,092,072,073,062,041,040$,
$5-051,050,042,037,109,091,052,107,089,070$,
$6-103,087,074,102,068,069,032,088,081,060$,
$7-059,022,024,027,048,033,125,126,096,066$,
$8-084,095,086,065,097,085,067,098,064,035$,
$9-124,082,094,058,023,123,028,046,122,047$,
$10-034,020,013,012,115,055,114,099,106,090$,
$11-056,104,014,015,112,057,113,105,021,029$,
$12-016,111,011,017,019,018$.

\section{REFERENCES}

1. K. S. Carman, J. C. Harden, and E. E. Posey, Appendix [to the authors' master's theses], University of Tennessee, about 1949. (The copy examined was reproduced at the University of Washington, 1954, $8 \mathrm{pp}$.)

2. National Bureau of Standards, Manual of the SWAC computing system, multilithed typescript, approx. 200 pages. [Available in limited quantity from Numerical Analysis Research, Department of Mathematics, University of California, Los Angeles 24, California.]

3. A. R. Poole, Finite ova, doctoral dissertation, California Institute of Technology, 1935 [not personally examined].

4. - Finite ova, Amer. J. Math. vol. 59 (1937) pp. 23-32.

5. H. S. Zuckerman and Edwin Hewitt, Notes on convolution algebras, hectographed typescript, University of Washington, about 1954, 11 pp.

University of California, Los Angeles 
-Nmmm-m-m-

स्दि - NONOOHm$000-m 000 \mathrm{mo}$ $00000000 \mathrm{mo}$

MMNNNOOONO مै 00000 मनमन 0000000000 0000000000

0000000000 स्य 0000000000 0000000000 0000000000

0000000000 జ्4 0000000000 0000000000 0000000000 mmm Nmmm Nmm O - fmolomomm $00 \mathrm{~mol} 000 \mathrm{~mm}$

OOONNNNmMO NNNNNNNNN $000000000 \mathrm{H}$ 0000000000

0000000000 0000000000 0000000000 0000000000

0000000000 0000000000 0000000000 0000000000
Nmmm NmNmmm NNmNmmNNm-

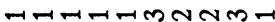
OOOOOMNNmO

NNNmmmNNN NNNNNNNNNO H-H-HनNNNO OOOOOONNNO

$000000000 \mathrm{~m}$ 0000000000 0000000000 000000000

0000000000 0000000000 0000000000 0000000000
" NNNONOONON H-WM-00-00 0000000000

mNNONOHNNN OO-NNNNNNN $00000 \mathrm{H}$ NN $00000000 \mathrm{~N}$

मनममनमनममन 0000000000 0000000000 0000000000

0000000000 0000000000 0000000000 0000000000

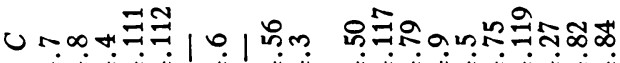

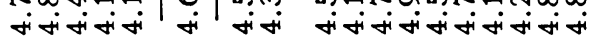

OnNm+un

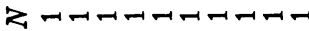
0000000000
Ondmanon a NNNNNNNNNN 0000000000

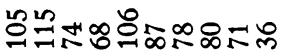

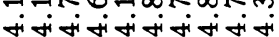

On NmALUR пмпмпмпmмm 0000000000

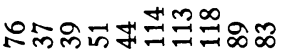

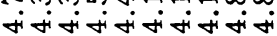

-

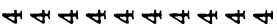
0000000000
2

2

शี

O-no

ठ००न

श्2

$000-$

0000

U Hamलंत्रत
omnNmNnNma OOOHNHONHA OOOONOONOO

$00000-00 \mathrm{H}$ 000000 HनH 0000000000

0000000000 0000000000 0000000000
NhNNOONH NNNNNNOO ONNNNHN

meNmNNOO m-m-0m0 N $000 \mathrm{O} 00 \mathrm{H}$

OODONNHN $000000 \mathrm{HA}$ 00000000

OHMOHMMMmO OOOHANHNmH $000000 \pi-1 m 0$ $00000000 \mathrm{mo}$

$000000000 \mathrm{H}$ 0000000000 0000000000 0000000000

0000000000 0000000000 0000000000 0000000000

0000000000 0000000000 0000000000 0000000000

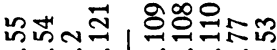
मitiqu

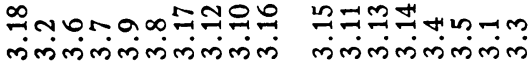

$z^{\text {OHNm }}$

onnmanon 0000000000
OANmHLON मनन-नमनन omnmtintora 0000000000 0000000000 
-n-mm-mmN2 mmmmm-mm-10 mmmnnNmmmn omommommon

mmnmm-nn-N مُ

H-NNNANHMN H-H-NNHNNN $000000000 \mathrm{~N}$

mmm

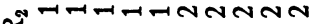

-П-म-न-म0000000000 0000000000 ब्र 0000000000 0000000000 0000000000

ง พธึั

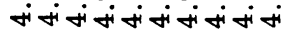

o-Nm+10 Zalaraaraa 0000000000 mmo00000000 nпмnm

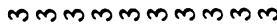
mmnnmmm nחm

Nतmmmmmmmm NNOHNNNNAN NNOOOHAOHन NNOOOOOOOO

mNHOOOOHOWH- $00000 \mathrm{CH}$ $0-00000000$

0 0000000000 0000000000 0000000000

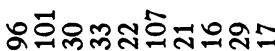

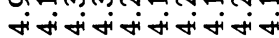

om 0000000000

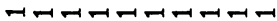

$000-000-1 m-$ mmolo $0000 \mathrm{mH}$ mッNNNmmmmmNNNNNNmm

mmolo Nmol NNNNNNNNON DONNNNNNON

mmNNNNmmmm HNNNNNNNmm $00000000 \mu-1$

mmNONNNNNN DONNNNNNNN o000000000 0000000000
NलO-O $0000-0$ mmoONN mm-nmm

$0000-10$ mmomom NतOmmm NNHFNN

mmol Non NNOmmm $0-0 m 00$ $00-m-1$

mmNNm-NN 00 000000

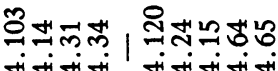
mกำ संमें मं

o-Nm+4 -r $-1-m-1-1$

0 mm NNNNNN H-H mmmm Nmm Nmm

2 NNmNNmmom

H-Nm-H-HOm

OONmOOODOM

NNNNNNNMOO

NNNNNNNNNN

NNNNH-H-OO

NNNNOOOOOO

H-HNH-H-O

स्द

0000 - 0 - 00

$00000000 \mathrm{H}$

0000000000

0000000000 F0000000000

\& 000000000

0000000000
Nmm Nmmmm-n NNmmmNmNOO

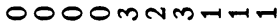
o $000 \mathrm{mmo} 00$

NNNmmNNNNN NNNNNNNNN - 000 NNONN OOOOONNONN

0000000 0000000000 मनमनममननमन 0000000000

0000000000 0000000000 0000000000 0000000000

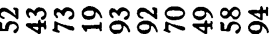
मं $\dot{+} \dot{+} \dot{+} \dot{+} \dot{+} \dot{+} \dot{4}$ mmmnNONOOM-m-N -mm-mmol

NNNNNNHmNNNNNN-F-H NNNNNN-HNNNNNOOOOO

$000000 \mathrm{OH}-1$

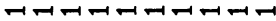
0000000000

0000000000 0000000000 0000000000 0000000000

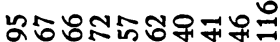

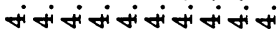

O NNNANNA 0000000000
mmmmNmm Nmm mN-MNNmmmm m0000000000

HNHANNNmMN HMNNNNNNN H-Hम-Hन-मN 0000000000

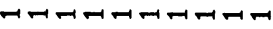
-

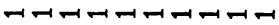
0000000000

0000000000 0000000000 0000000000 0000000000

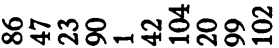

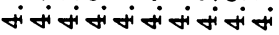

$\infty \infty \infty \infty \infty \infty \infty \infty \infty$ 0000000000
ח 0000000000 ondmantora 0000000000 0000000000 